\title{
Syncope, CTCAE 5.0
}

National Cancer Institute

\section{Source}

National Cancer Institute. Syncope, CT CAE 5.0. NCI Thesaurus. Code C146750.

A disorder characterized by spontaneous loss of consciousness caused by insufficient blood supply to the brain. 